\title{
Kinetic Inductance of Josephson Junction Arrays: Dynamic and Equilibrium Calculations
}

\author{
Wenbin Yu and D. Stroud \\ Department of Physics, The Ohio State University, Columbus, OH 43210
}

(October 27, 2018)

\begin{abstract}
We show analytically that the inverse kinetic inductance $L^{-1}$ of an overdamped junction array at low frequencies is proportional to the admittance of an inhomogeneous equivalent impedance network. The $i j^{\text {th }}$ bond in this equivalent network has an inverse inductance $J_{i j} \cos \left(\theta_{i}^{0}-\theta_{j}^{0}-A_{i j}\right)$, where $J_{i j}$ is the Josephson coupling energy of the $i j^{\text {th }}$ bond, $\theta_{i}^{0}$ is the ground-state phase of the grain $i$, and $A_{i j}$ is the usual magnetic phase factor. We use this theorem to calculate $L^{-1}$ for square arrays as large as $180 \times 180$. The calculated $L^{-1}$ is in very good agreement with the low-temperature limit of the helicity modulus $\gamma$ calculated by conventional equilibrium Monte Carlo techniques. However, the finite temperature structure of $\gamma$, as a function of magnetic field, is sharper than the zero-temperature $L^{-1}$, which shows surprisingly weak structure. In triangular arrays, the equilibrium calculation of $\gamma$ yields a series of peaks at frustrations $f=\frac{1}{2}(1-1 / N)$, where $N$ is an integer $\geq 2$, consistent with experiment.
\end{abstract}

PACS numbers: 74.50.+r, 74.60.Ge, 74.60.Jg, 74.70.Mq 


\section{INTRODUCTION.}

Superconducting arrays have been a subject of continuing interest for a number of years [1]. Such arrays typically consist of a large collection of superconducting grains, of micron or submicron dimensions, arranged in a periodic structure. They are embedded in a nonsuperconducting host which mediates a Josephson or proximity effect coupling between the grains. The coupling generally produces a transition to a coherent state at a temperature $T_{c}(B)$ which is well below the superconducting transition temperature $T_{c 0}$ of the individual grains, and which strongly depends on the applied magnetic field $B$. There has been extensive research on the thermodynamic properties of these arrays, especially as a function of $B$ [2 8]. More recently, a number of workers have extended these studies to the dynamical response of such arrays, especially their a. c. $I V$ characteristics [9] and vortex motions in the array $[10$.

In theoretical studies, the thermodynamic response of superconducting arrays is commonly characterized by the magnetic field and temperature dependent helicity modulus, $\gamma(B, T) . \gamma$ is defined as the stiffness of the array to a twist in the phase of the superconducting order parameter [3 5]. It is thus closely analogous to the spin wave stiffness in a magnetic material. $\gamma$ can readily be expressed as an equilibrium quantity and evaluated as a thermodynamic average in the canonical ensemble, e. g., by Monte Carlo simulation. On the other hand, the measurement of $\gamma$ is typically a dynamical problem [11,12]. Such a measurement is based on the identification of $\gamma$ with the inverse kinetic inductance $L^{-1}$, where $L$ is the effective inductance of the array. $L$ can be measured at temperatures below $T_{c}(B)$ by applying an appropriate a. c. current and measuring the out-of-phase part of the a. c. voltage response. Thus, in order to compare theory and experiment, one usually equates a thermodynamic quantity to a dynamical one.

In this paper, we describe a straightforward dynamical procedure for calculating $\gamma$ in a superconducting array at temperature $T=0$. The method starts from the coupled Josephson equations for the array in the limit of low frequency and small amplitude. By carrying out an appropriate Taylor expansion in this limit, we show that $\gamma$ is equivalent to the inverse inductance of a certain inhomogeneous impedance network. The impedances in this network are determined by the ground-state phases arrangement in the array. Once the ground state is known, the effective impedance of the network can be calculated by standard numerical, or even analytical, techniques.

To show that this method is practical, we apply it to a square Josephson-coupled network subjected to an applied transverse magnetic field. The resulting $\gamma(T=0)$ has surprisingly weak structure as a function of magnetic field. Remarkably, the structure appears most conspicuously at finite temperature, because $T_{c}(B)$ is an extremely sensitive function of

$B$. To verify the consistency of this picture, we calculate $\gamma$ by the standard equilibrium 
approach. We show that, for all values of $B$ considered, it approaches the $T=0$ value obtained by dynamical techniques.

We have also used the equilibrium approach to calculate $\gamma(B, T)$ for a triangular lattice as a function of magnetic field. The dynamical approach is still applicable, in principle, to triangular lattices. However, we have not used it in this case, because our algorithm for computing impedances does not always converge for triangular lattices. Our calculated equilibrium $\gamma(B, T)$ has a very rich structure, as a function of $B$, which agrees in some detail with that found in recent dynamical measurements by Théron et al [12]. This agreement confirms, once again, that the static and dynamical quantities are basically equivalent, and also shows that the array is well described by the standard frustrated XY model which underlies both the static and dynamic theories.

We turn now to the body of the paper. Section II describes the formalism underlying our calculations. In Section IIA, we prove the equivalence of $\gamma(T=0)$ to the effective impedance of an inhomogeneous impedance network. Section IIB reviews the standard equilibrium method for calculating $\gamma$. Section III describes our numerical method for calculating both the effective impedance and the quantities needed for an equilibrium evaluation of $\gamma(T)$. Our results for both square and triangular lattices are presented in Section IV. A brief discussion follows in Section V.

\section{FORMALISM.}

Our basic geometry is shown in Fig. 1. It consists of a periodic network of superconducting grains, each of which is Josephson coupled to its nearest neighbors. We can calculate the inverse kinetic inductance $L^{-1}$, of this network by two different methods. In the first method, we use an identity, proven below, which shows that $L^{-1}$ is equivalent, within a proportionality constant, to the admittance of an inhomogeneous equivalent network. This identity permits calculation of $L^{-1}$, or equivalently the helicity modulus $\gamma$, at temperature $T=0$. The second method is particularly appropriate at finite temperatures. It uses the fact that $\gamma$ is an equilibrium thermodynamic quantity which can be calculated by standard Monte Carlo techniques. We review this type of calculation in Section IIB below.

\section{A. Helicity Modulus at $T=0$ : Dynamical Evaluation.}

We consider an overdamped resistively-shunted Josephson junction (RSJ) array in the presence of an external magnetic field $\mathbf{B}$ applied perpendicular to the array. The dynamical equations for the array at zero temperature take the form

$$
I_{i j}=\frac{V_{i j}}{R_{i j}}+I_{c ; i j} \sin \left(\theta_{i}-\theta_{j}-A_{i j}\right),
$$




$$
\begin{aligned}
V_{i j} & =\frac{\hbar}{2 e} \frac{d}{d t}\left(\theta_{i}-\theta_{j}\right), \\
\sum_{j} I_{i j} & =I_{i ; \text { ext }} .
\end{aligned}
$$

Here $I_{i j}$ is the current from grain $i$ to grain $j, V_{i j} \equiv V_{i}-V_{j}$ is the voltage difference between grains $i$ and $j, I_{c ; i j}$ and $R_{i j}$ are the critical current and shunt resistance of the $i j^{\text {th }}$ junction, $\theta_{i}$ is the phase of the order parameter on the $i^{t h}$ grain, and $I_{i \text {;ext }}$ is the external current fed into the $i^{t h}$ grain. $A_{i j}$ is a magnetic phase factor defined by the relation

$$
A_{i j}=\frac{2 \pi}{\Phi_{0}} \int_{i}^{j} \mathbf{A} \cdot \mathbf{d l}
$$

where $\mathbf{A}$ is the vector potential for the external magnetic field, and $\Phi_{0}=h c / 2 e$ is the flux quantum. Eq. (1) simply expresses the total current of each junction as the sum of a normal current through a shunt resistance and a Josephson supercurrent. Eq. (2) is the Josephson relation connecting the voltage drop across a junction to the phase difference. Eq. (3) is just the Kirchhoff's Law, expressing the current conservation at the $i^{\text {th }}$ grain. In the calculations below, we will assume that the vector potential is that of the externally applied magnetic field. This is equivalent to assuming that the intergranular Josephson coupling is so weak that the screening magnetic fields can be neglected.

Let us assume that in the absence of an external current, the $i^{\text {th }}$ phase has a value of $\theta_{i}^{0}$. We assume that the collection of phases $\left\{\theta_{i}^{0}\right\}$ represents a local energy minimum. One possible state of this kind is the true ground state of the array, but there may be other metastable states which also satisfy this condition. We now consider an a. c. external current $I_{i \text {;ext }}=\operatorname{Re}\left[I_{i 0} \exp (-i \omega t)\right]$, where $I_{i 0}$ is an appropriate amplitude. If the amplitude is not too large, the induced voltages and phase changes will also be small, and we may assume that they also have frequency $\omega$. Hence, we write the phase on the $i^{\text {th }}$ grain as

$$
\theta_{i}(t)=\theta_{i}^{0}+\delta \theta_{i}(t)
$$

We can now use these assumptions to linearize the equations of motion about the state $\left\{\theta_{i}^{0}\right\}$. Combining Eqs. (1) - (3), we can write

$$
\sum_{j}\left(I_{c ; i j} \sin \left(\theta_{i}^{0}-\theta_{j}^{0}-A_{i j}+\delta \theta_{i}-\delta \theta_{j}\right)+\frac{\hbar\left(\dot{\delta} \theta_{i}-\dot{\delta} \theta_{j}\right)}{2 e R_{i j}}\right)=I_{i ; e x t} .
$$

Expanding the sine function in a Taylor series to first order in $\delta \theta_{i}-\delta \theta_{j}$, and using the fact that in the absence of external current, the state $\left\{\theta_{i}^{0}\right\}$ satisfies

$$
\sum_{j} I_{c ; i j} \sin \left(\theta_{i}^{0}-\theta_{j}^{0}-A_{i j}\right)=0,
$$

we obtain the simple relation 


$$
\sum_{j}\left[\left(\delta \theta_{i}-\delta \theta_{j}\right) I_{c ; i j} \cos \left(\theta_{i}^{0}-\theta_{j}^{0}-A_{i j}\right)+\frac{\hbar\left(\dot{\delta \theta_{i}}-\dot{\delta} \theta_{j}\right)}{2 e R_{i j}}\right]=I_{i ; e x t} .
$$

Finally, we use the assumption that $\delta \theta_{i}$ is varying sinusoidally in time with frequency $\omega$ to rewrite this equation as

$$
\sum_{j}\left[\frac{2 i e}{\hbar \omega} I_{c ; i j} \cos \left(\theta_{i}^{0}-\theta_{j}^{0}-A_{i j}\right)+\frac{1}{R_{i j}}\right] V_{i j}=I_{i ; e x t},
$$

where we have used the relation

$$
V_{i j}=\frac{\hbar}{2 e}\left(\dot{\delta \theta_{i}}-\dot{\delta} \theta_{j}\right)=-i \frac{\hbar \omega}{2 e}\left(\delta \theta_{i}-\delta \theta_{j}\right) .
$$

We can interpret equations (7) in a very simple way. Namely, they are identical to those for an inhomogeneous impedance network responding to the driving a. c. current. The $i j^{\text {th }}$ admittance is the sum of two terms in parallel: a conductance $g_{i j}=1 / R_{i j}$, and a purely imaginary inductive element $[2 i e /(\hbar \omega)] I_{c ; i j} \cos \left(\theta_{i}^{0}-\theta_{j}^{0}-A_{i j}\right)$. The elements of this network are all known if the unperturbed metastable state $\left\{\theta_{i}^{0}\right\}$ is known at the fields of interest. Given the elements of the network, the effective network admittance can be obtained by standard numerical techniques which have been developed for finding the admittances of inhomogeneous impedance networks [13, 14].

The results are particularly simple in the limit of low frequency. In this case, the shunt resistances are irrelevant, and the response of the network is purely inductive. The effective inductance of the network is then often known as the kinetic inductance. The present work permits this kinetic inductance to be computed dynamically, at least at $T=0$, by solving for the impedance of an effective impedance network. A numerical technique for carrying out this calculation is summarized below.

\section{B. Helicity Modulus at Finite Temperature: Equilibrium Evaluation.}

The kinetic inductance of an overdamped Josephson network can also be calculated as an equilibrium quantity, by connecting it to the so-called helicity modulus of the network. This

connection has been known for more than ten years [3], but for completeness we summarize this relation in the following paragraphs.

The equilibrium Hamiltonian of the Josephson network is given by

$$
H=-\sum_{i j} E_{i j} \cos \left(\theta_{i}-\theta_{j}-A_{i j}\right)
$$

where $E_{i j}=\hbar I_{c ; i j} /(2 e)$ is the coupling energy between grains $i$ and $j$, and $A_{i j}$ is given by Eq. (4). In our calculations, we assume that $E_{i j}$ is the same for each junction. The equilibrium thermodynamic properties are given by the free energy 


$$
\mathcal{F}=-k_{B} T \ln Q_{N}(T)
$$

where the $N$-particle partition function $Q_{N}$ is given by the canonical integral

$$
Q_{N}(T)=\int_{0}^{2 \pi} \cdots \int_{0}^{2 \pi} \Pi_{i} d \theta_{i} \exp \left(-H / k_{B} T\right)
$$

Similarly, the canonical average of any desired function $\mathcal{O}\left(\theta_{1}, \cdots, \theta_{N}\right)$ is given by

$$
<\mathcal{O}>=\frac{1}{Q_{N}} \int \cdots \int \Pi_{i} d \theta_{i} \mathcal{O}\left(\theta_{1}, \cdots, \theta_{N}\right) \exp \left(-H / k_{B} T\right)
$$

The helicity modulus $\gamma_{\alpha \beta}$ (or equivalently, the superfluid density) is defined as the free energy cost of imposing a twist in the phase at the boundaries of the sample. The principal elements of $\gamma_{\alpha \beta}$ can be thought of as spin-wave stiffness constants appropriate to "phase waves" in this weakly coupled system. Rather than imposing a twisted boundary condition and calculating the resulting increase in free energy, however, it is more convenient to use periodic boundary conditions and calculate $\gamma_{\alpha \beta}$ as

$$
\gamma_{\alpha \beta}=\left(\frac{\partial^{2} \mathcal{F}}{\partial A_{\alpha}^{\prime} \partial A_{\beta}^{\prime}}\right)_{\overrightarrow{A^{\prime}=0}} .
$$

Here $\overrightarrow{A^{\prime}}$ represents an added uniform vector potential (in addition to that which produces the applied magnetic field) which is included in the Hamiltonian in order to produce a twist. The various second derivatives in Eq. (13) are readily computed for an ordered or a disordered sample, with the result for, e. g., $\gamma_{x x}$ :

$$
\begin{aligned}
N \gamma_{x x} & =\left\langle\sum_{<i j>} E_{i j} x_{i j}^{2} \cos \left(\theta_{i}-\theta_{j}-A_{i j}\right)\right\rangle \\
& -\frac{1}{k_{B} T}\left[\left\langle\left(\sum_{<i j>} E_{i j} x_{i j} \sin \left(\theta_{i}-\theta_{j}-A_{i j}\right)\right)^{2}\right\rangle\right] \\
& +\frac{1}{k_{B} T}\left[\left\langle\sum_{<i j>} E_{i j} x_{i j} \sin \left(\theta_{i}-\theta_{j}-A_{i j}\right)\right\rangle^{2}\right]
\end{aligned}
$$

where $x_{i j}=x_{j}-x_{i}$ and $x_{i}$ is the $x$ coordinate of the center of grain $i$. Similar expressions hold for the other components of $\gamma$. The above expressions are valid for both square and triangular lattices.

We now connect $\gamma_{\alpha \beta}$ to the inductive response of the array. To make this connection, note that the first derivative of the free energy is the local current density $\mathbf{J}$, i. e.,

$$
\left(\frac{\partial \mathcal{F}}{\partial A_{\alpha}^{\prime}}\right)_{\vec{A}^{\prime}=0}=-J_{\alpha} / c .
$$

Hence, 


$$
\gamma_{\alpha \beta}=-\frac{1}{c}\left(\frac{\partial J_{\alpha}}{\partial A_{\beta}^{\prime}}\right)_{\overrightarrow{A^{\prime}=0}} .
$$

If $\overrightarrow{A^{\prime}}$ is produced by an a. c. electric field $\vec{E}$ of frequency $\omega$, then $\overrightarrow{A^{\prime}}=-i c \vec{E} / \omega$. If we introduce the conductivity $\sigma_{\alpha \beta}$ by $\sigma_{\alpha \beta} \equiv\left(\partial J_{\alpha} / \partial E_{\beta}\right)_{\vec{E}=0}$, then it follows that $\gamma_{\alpha \beta}=-i \omega \sigma_{\alpha \beta} / c^{2}$. At very low frequencies, $\sigma_{\alpha \beta}$ is of the purely inductive form $\sigma_{\alpha \beta}=i L_{\alpha \beta}^{-1} / \omega$, where $L_{\alpha \beta}^{-1}=c^{2} \gamma_{\alpha \beta}$. Thus $\gamma_{\alpha \beta}$ is indeed proportional to low-frequency inductive response $L_{\alpha \beta}^{-1}$ of the array.

In general, $\gamma_{\alpha \beta}$ is a tensor, not a scalar. In square or triangular arrays, however, the fourfold or sixfold rotational symmetry implies that $\gamma_{\alpha \beta}$ is, in fact, just a multiple of the unit tensor, i. e. $\gamma_{\alpha \beta}(T)=\gamma(T) \delta_{\alpha \beta}$.

\section{METHOD OF CALCULATION.}

In all the calculations in this paper, we assume that the array is homogeneous and ordered. That is, we assume that the coefficients $I_{c ; i j}$ and $R_{i j}$ are independent of position and equal to $I_{c}$ and $R$ respectively. It follows that the coupling energies $E_{i j}$ are also positionindependent and equal to a constant which we denote simply as $E_{J}=\hbar I_{c} /(2 e)$.

\section{A. Effective Impedance.}

For the impedance calculation, we inject a uniform a. c. current $I_{\text {ext }}$ into each grain at one boundary and extract the same current from each grain in the opposite boundary. To obtain the kinetic inductance by the method of Section IIA, we need to compute the effective impedance of a certain equivalent network. The inverse inductance of the $i j^{\text {th }}$ bond in this network is $I_{c} \cos \left(\theta_{i}^{0}-\theta_{j}^{0}-A_{i j}\right)$, where $\theta_{i}^{0}$ denotes the ground-state phase of the $i^{\text {th }}$ grain. Thus, the problem divides into two parts: (i) finding the ground-state configuration $\left\{\theta_{i}^{0}\right\}$ in the absence of current; and (ii), given that configuration, computing the effective impedance of the network. In practice, we cannot be assured that we have obtained the true ground state; in general, we will obtain a metastable configuration which may be close to the ground state.

To obtain a metastable state near the ground state phase configuration, we carry out a standard Monte Carlo annealing [15] at fixed magnetic field, starting from a high temperature and cooling gradually. The purpose of this annealing is to avoid, or at least to minimize, the trapping into metastable states which is a notorious consequence of dealing with frustrated systems, such as these arrays. In this part of the calculation, periodic boundary conditions are used in all directions. To check that we are indeed dealing with a near-ground-state configuration, we generally carry out more than 50 anneals at a given temperature. The effective impedance is computed for the state with the lowest energy. 
To calculate the effective impedance of the network, we need to solve the set of sparse linear equations (7). In practice, at the low-frequency limit, the shunt resistance contribution can be discarded, and the trivial common factor of $i / \omega$ divided out. For both square and triangular arrays, we then use the Gauss-Seidel iterative method to solve for the impedance of networks as large as $180 \times 180$. This iterative procedure always converges in our square arrays calculations. In our triangular array calculations, the iterative approach sometimes fails to converge, possibly because the ground state factors $\cos \left(\theta_{i}^{0}-\theta_{j}^{0}-A_{i j}\right)$ can be of both signs for triangular arrays. Thus, we will present results for triangular arrays using only the equilibrium method described above.

\section{B. Helicity Modulus at Finite Temperature.}

To calculate the helicity modulus $\gamma_{\alpha \beta}$ at finite temperature, we use the equilibrium Monte Carlo method within the standard Metropolis algorithm [16] for both square and triangular arrays with periodic boundary conditions. In order to minimize the effects of metastable states, we cooled our system from high temperatures at each magnetic field, typically starting from $1.5 E_{J} / k_{B}$ and cooling to $0.5 E_{J} / k_{B}$ in steps of $0.1 E_{J} / k_{B}$, followed by an additional cooling down to $0.2 E_{J} / k_{B}$ in steps of $0.05 E_{J} / k_{B}$. Following this cooling, the required averages were obtained by making 60000 passes through the entire lattices, with the first 10000 passes discarded. We carry out the thermal average by including only every tenth pass through the entire array, in order to minimize the strong statistical correlation between successive configurations. The reported results are averages of $\gamma=\left(\gamma_{x x}+\gamma_{y y}\right) / 2$ and are generally produced by averaging over four to eight independent runs.

\section{RESULTS.}

\section{A. Square Arrays.}

Fig. 2 shows the helicity modulus $\gamma$ plotted as a function of frustration values $f$ at four different temperatures in square arrays. The array sizes are either $48 \times 48$ or $50 \times 50$ in the finite-temperature calculations, which are carried out by the equilibrium technique of Section IIB. In the $T=0$ calculations, we use arrays from $120 \times 120$ to $180 \times 180$ and the analog technique of Section IIA. The frustration $f \equiv \Phi / \Phi_{0}$, where $\Phi$ is the flux per plaquette. Note that as temperature decreases, these Monte Carlo calculations converge remarkably well to the $T=0$ impedance results. This supports the validity of our method for calculating the kinetic inductance at temperature $T=0$.

For $f=0$ and $f=1 / 2$, the melting temperature is known to equal approximately $0.95 E_{J} / k_{B}$ and $0.45 E_{J} / k_{B}$ respectively [2,3]. For $f=p / q$, with $p$ and $q$ mutually prime 
integers, as $q$ increases, the melting temperature, estimated as the point where $\gamma$ goes to zero, decreases very quickly, consistent with previous work [3]. At $f=5 / 12$, for example, the melting temperature is probably less than $0.1 E_{J} / k_{B}$ (the vanishing of $\gamma$ at this value of $f$ is considerably broadened by finite size effects).

A remarkable feature of the results shown in Fig. 2 is that the plot of $\gamma(f)$ becomes sharper as the temperature is increased. This sharpening with increasing temperature is seemingly contrary to intuition, but in fact is easily explained. As noted above, the melting temperature of the vortex lattice at a frustration $f=p / q$ decreases with increasing $q$ (assuming $p$ and $q$ mutually prime integers). Thus, at extremely low temperatures, the vortex lattice is stable for a wider range of $f$ values, but at slightly higher temperature, it has melted at all except a few values of $f$ corresponding to relatively small values of $q$. The corresponding $\gamma(f)$ shows sharp peaks only at a few such values of $f$. (As seen in Fig. 2, the sharpest features at a temperature of $0.1 E_{J} / k_{B}$ occur at $f=1 / 5,1 / 3,2 / 5$, and $1 / 2$.) This explains why the zero-temperature $\gamma(f)$ is surprisingly featureless: for all values of $f$, the vortex lattice is apparently frozen at $T=0$.

It should be emphasized that the $T=0$ calculations are carried out using "ground states" obtained by Monte Carlo annealing from a finite temperature. As pointed out in the previous section, such annealing is not guaranteed to produce the true ground state, but only a metastable state close to the ground state. We have checked for metastability by calculating $\gamma$ at $T=0$ using several such annealed states. The resulting $\gamma$ 's are generally quite close to that obtained in the state of lowest energy. The $\gamma$ 's plotted in Fig. 2 always correspond to this lowest energy state.

\section{B. Triangular Arrays.}

Fig. 3 shows the calculated helicity modulus $\gamma$, plotted as a function of the frustration value $f$ in a triangular array at four different temperatures. As in a square array, when $f=p / q$, where $p$ and $q$ are mutually prime integers, the melting temperature tends to decrease strikingly with increasing $q$. Indeed, when $q$ is sufficiently large (typically, when $q \geq 8$ ), the melting temperature of the array is less than $\left.0.1 E_{J} / k_{B}\right)$. As in the square array, there are a series of sharp peaks in $\gamma(f, T)$ plotted as a function of $f$ at fixed $T$. Some of this structure has been previously studied [4], but the present work represents a considerably more detailed investigation. We have found clearly distinguishable peaks in $\gamma$ at frustration values $f=\frac{1}{2}, \frac{1}{3}, \frac{1}{4}, \frac{2}{5}, \frac{3}{7}, \frac{3}{8}, \cdots$. Indeed, we have obtained peaks at every value of $f$ between $1 / 3$ and $1 / 2$ satisfying the relation $f=\frac{1}{2}(1-1 / N)$, with $\mathrm{N}$ as large as 8 . All such peaks have been observed in recent experiments by Théron et al [12]. Since we are able to obtain such peaks here, we conclude that the experimental arrays are adequately described by a frustrated XY model, as assumed here. Of course, in the calculations, we are unable to vary 
$f$ continuously as is possible in the experimental geometry. Thus, we are able to identify "peaks" only by considering nearby values of $f$ not satisfying the relation $f=\frac{1}{2}(1-1 / N)$, and showing that these have smaller values of $\gamma$ than the $f$ values in the chosen series. We have shown a few such values in Fig. 4. As one illustration, the value of $\gamma$ at $f=3 / 8$ are larger than those at $f=17 / 48$ and $f=19 / 48$.

Théron et al [12] account for the peaks at $f=\frac{1}{2}(1-1 / N)$ on the basis of a superlattice of vacancies in a background of the well-known checkerboard vortex ground state which forms at $f=\frac{1}{2}$. In their model, a change in the nature of the ground state is predicted at a critical frustration $f_{c} \approx 0.468$. When $f_{c}<f<\frac{1}{2}$, a $2 \mathrm{D}$ vacancy superlattice is found to be energetically favored, while for $1 / 3<f<f_{c}$, a vortex superlattice consisting of 1D parallel stripes is preferred.

In order to check this conjecture in our simulation, we have calculated the lowtemperature vortex configuration of the vortex lattice at various values of $f$. In a Josephson junction array with frustration $f$, the vortex number $n$ of each plaquette is defined as:

$$
\sum_{\text {plaquette }}\left(\theta_{i}-\theta_{j}-A_{i j}\right)+2 \pi f=2 \pi n
$$

where the gauge invariant phase difference for each junction $\left(\theta_{i}-\theta_{j}-A_{i j}\right)$ is in the range $(-\pi, \pi]$ and the summation is taken in the counterclockwise direction.

Some of our calculated vortex configurations are shown in Fig. 4. Fig. 4 (a) shows the configuration at $f=3 / 8=0.375$ at temperature $T=0.01 E_{J} / k_{B}$. It is easy to see there is indeed a striped domain structure in the vortex lattice, although the domain wall configuration is somewhat different from that predicted by [12]. For lattices such that $f>f_{c}$, it is necessary to carry out the calculations for relatively large lattices in order to distinguish the results from the $f=1 / 2$ case. It proves very difficult to observe the predicted vacancy lattice structure. Fig. 4 (b) shows a representative low-temperature vortex configuration for an $f$ value in this range, namely $f=17 / 36=0.472$ at a temperature $T=0.01 E_{J} / k_{B}$. It is difficult to categorize the vortex lattice into any of the domain patterns suggested by [12]. Nevertheless, we do obtain nearly all the peaks found experimentally, at most of the fields studied. We conclude that, while the experimental peaks do correspond to particularly stable vortex structures, there are many structures at these frustrations which are nearly equally stable, and which could all give rise to the observed peaks.

Finally, we have checked for hysteresis at $f=1 / 6$ in order to test whether the melting transition at that field is first-order. In $18 \times 18$ triangular arrays, we have used a Monte Carlo annealing method first to cool the array from a temperature $1.5 E_{J} / k_{B}$ to $0.1 E_{J} / k_{B}$ gradually, and then to reheat it. We find a clear hysteresis in the energy in the temperature range between $0.25 E_{J} / k_{B}$ and $0.32 E_{J} / k_{B}$ [cf. Fig. 5], suggestive of a first-order transition. This result is consistent with expectations based on three-dimensional calculations on stacked triangular lattices by Hetzel et al [17], at $f=1 / 6$. 


\section{CONCLUSION.}

In this paper, we have developed a new method to calculate the kinetic inductance of a Josephson junction array at zero temperature. This method maps a junction array onto a linear complex impedance network. The mapping permits us to calculate the zerotemperature kinetic inductance using much larger arrays than previously possible, provided that the ground state can be obtained by other means. The kinetic inductance calculated by this method agrees well with Monte Carlo calculations of the helicity modulus, in the low temperature limit. In both square and triangular arrays, the helicity modulus shows a series of peaks as a function of the frustration $f$. In triangular arrays, these peaks occur at $f=\frac{1}{2}(1-1 / N)$, where $N$ is an integer $\geq 2$, in agreement with experiment.

The dynamical method discussed here has other potential applications. For example, it can be extended to treat arrays at finite frequencies, i. e., to study the variation of the effective admittance with frequency, simply by retaining the shunt resistances in Eq. (7). It can also be used with little change to treat underdamped arrays. Finally, it may be possible to extend this approach to finite temperatures, by including a Langevin noise term in the equations of motion for the phases.

\section{ACKNOWLEDGMENTS.}

We are grateful to R. Šášik and X. Zhang for valuable conversations. This work has been supported by NSF grant DMR90-20994, and by the Midwest Superconductivity Consortium at Purdue University through D. O. E. grant DE-FG90-02-ER-45427. Calculations were carried out, in part, on the CRAY Y-MP 8/8-64 of the Ohio Supercomputer Center. 


\section{REFERENCES}

[1] For many references up to 1988, see, e. g., the articles in Physica (Amsterdam) 152B, pp. 1-302 (1988). Some more recent reviews, may be found in Proceedings of the 2nd CTP Workshop on Statistical Physics: KT Transition and Superconducting Arrays, edited by D. Kim, J. S. Chung, and M. Y. Choi (Min Eum Sa, Seoul, Korea, 1993).

[2] J. Tobochnik and G. V. Chester, Phys. Rev. B 20, 3761 (1979).

[3] S. Teitel and C. Jayaprakash, Phys. Rev. B 27, 598 (1983); S. Teitel and C. Jayaprakash, Phys. Rev. Lett. 51, 1999 (1983).

[4] W. Y. Shih and D. Stroud, Phys. Rev. B 30, 6774 (1984).

[5] W. Y. Shih, C. Ebner and D. Stroud, Phys. Rev. B 34, 134 (1984).

[6] M. Y. Choi and S. Doniach, Phys. Rev. B 31, 4516 (1985).

[7] M. Yosefin and E. Domany, Phys. Rev. B 32, 1778 (1985).

[8] B. Berge, H. T. Diep, A. Ghazali and P. Lallemand, Phys. Rev. B 34, 3177 (1986).

[9] Interest in the a. c. response has focused especially on the occurrence of Shapiro steps in Josephson arrays. Some representative works are: S. P. Benz, M. Rzchowski, M. Tinkham, C. J. Lobb, Phys. Rev. Lett. 64, 693 (1990); K. H. Lee, D. Stroud, and J. S. Chung, Phys. Rev. Lett. 64, 962 (1990). D. Domínguez and J. V. José, Phys. Rev. Lett. 69, 514 (1992). L. L. Sohn, M. S. Rzchowski, J. U. Free, and M. Tinkham, Phys. Rev. B 45, 3003 (1992); D. Domínguez and H. A. Cerdeira, Phys. Rev. Lett. 71, 23359 (1993).

[10] For some recent studies of vortex dynamics in Josephson junction arrays, see, e. g., U. Eckern and A. Schmid, Phys. Rev. B 39, 6441 (1989); M. S. Rzchowski, S. P. Benz, M. Tinkham and C. J. Lobb, Phys. Rev. B 42, 2041 (1990); T. P. Orlando, J. E. Mooij and H. S. J. van der Zant, Phys. Rev. B 43, 10218 (1991); T. S. Tighe, A. T. Johnson, and M. Tinkham, Phys. Rev. B 44, 10286 (1991); R. Fazio and G. Schön, Phys. Rev. B 43, 5307 (1991); P. A. Bobbert, Phys. Rev. B 45, 7540 (1992); H. S. J. van der Zant, F. C. Fritschy, T. P. Orlando and J. E. Mooij, Europhys. Lett. 18, 343 (1992); U. Eckern and E. B. Sonin, Phys. Rev. B 47, 505 (1993); H. S. J. van der Zant, F. C. Fritschy, T. P. Orlando and J. E. Mooij, Phys. Rev. B 47, 295 (1993); U. Geigenmüller, C. J. Lobb, C. B. Whan, Phys. Rev. B 47, 348 (1993); W. Yu, K. H. Lee and D. Stroud, Phys. Rev. B 47, 5906 (1993); W. Yu and D. Stroud, Phys. Rev. B 49, 6174 (1994); T. J. Hagenaars, P. H. E. Tiesinga, J. E. van Himbergen, and J. V. José, to be published; 
[11] B. Jeanneret, J. L. Gavilano, G. A. Racine, Ch. Leemann and P. Martinoli, Appl. Phys. Lett. 55, 2336 (1989).

[12] R. Théron, J. -B. Simond, Ch. Leemann, H. Beck, P. Martinoli, and P. Minnhagen, Phys. Rev. Lett. 71, 1246 (1993); R. Théron, S. E. Korshunov, J. B. Simond, Ch. Leemann, and P. Martinoli, Phys. Rev. Lett. 72, 562 (1994).

[13] S. Kirkpatrick, Rev. Mod. Phys. 45, 574 (1973).

[14] D. J. Frank and C. J. Lobb, Phys. Rev. B 37, 302 (1988).

[15] See, for example, T. C. Halsey, Phys. Rev. B 31, 5728 (1985).

[16] K. Binder and D. W. Heermann, Monte Carlo Simulation in Statistical Physics, An Introduction, 2nd edition, Springer-Verlag, 1992.

[17] R. E. Hetzel, A. Sudbo, and D. A. Huse, Phys. Rev. Lett. 69, 518 (1992). 


\section{Figure Captions}

1. Schematic diagrams of (a) an $8 \times 8$ square and (b) an $8 \times 8$ triangular Josephson junction array. Each intersection represents a superconducting grain, which is connected to its nearest neighbors by Josephson coupling. In the dynamical inductance calculation, the external current is injected into each grain at one boundary and extracted from the other side boundary; free boundary conditions are used in the direction of current injection, while periodic boundary conditions are used in the transverse direction. In the Monte Carlo calculations, periodic boundary conditions are applied in both directions.

2. Calculated helicity modulus versus frustration $f$ at four different temperatures in square arrays. Lines merely serve to guide the eye. For clarity, the curves for $T=0,0.01 E_{J} / k_{B}$ and $0.05 E_{J} / k_{B}$ are shifted vertically by $0.3,0.2$, and 0.1 units respectively. The zero temperature result is obtained from a dynamical calculation of the inverse array inductance, using array sizes ranging from $120 \times 120$ to $180 \times 180$. The finite temperature results are obtained by Monte Carlo methods in $50 \times 50$ arrays for $f=1 / 5,2 / 5$, and in $48 \times 48$ arrays at all other values of $f$ shown.

3. Calculated helicity modulus versus frustration $f$ at four different temperatures in triangular arrays, as obtained by Monte Carlo simulations. Lines merely serve to guide the eye. For clarity, we have vertically shifted the plots for $T=0.01 E_{J} / k_{B}, 0.05 E_{J} / k_{B}$ and $0.1 E_{J} / k_{B}$ by by $0.3,0.2$, and 0.1 units respectively. The array sizes are $56 \times 56$ for $f=3 / 7 ; 50 \times 50$ for $f=1 / 5$ and $2 / 5$; and $48 \times 48$ for all other $f$ values shown.

4. Vortex configurations for triangular arrays as obtained by Monte Carlo annealing at temperature $T=0.01 E_{J} / k_{B}$ at two different values of $f:$ (a) $f=3 / 8,32 \times 32$ array (the striped pattern can be seen by viewing the array from lower right to upper left); (b) $f=17 / 36,36 \times 36$ array. Dark and light plaquettes denote the presence and absence of vortices.

5. Energy per grain in $18 \times 18$ triangular arrays at $f=1 / 6$, as calculated on cooling (solid disks) and on heating (open disks). The displayed results represent averages over five independent runs; lines serve to guide the eye. 


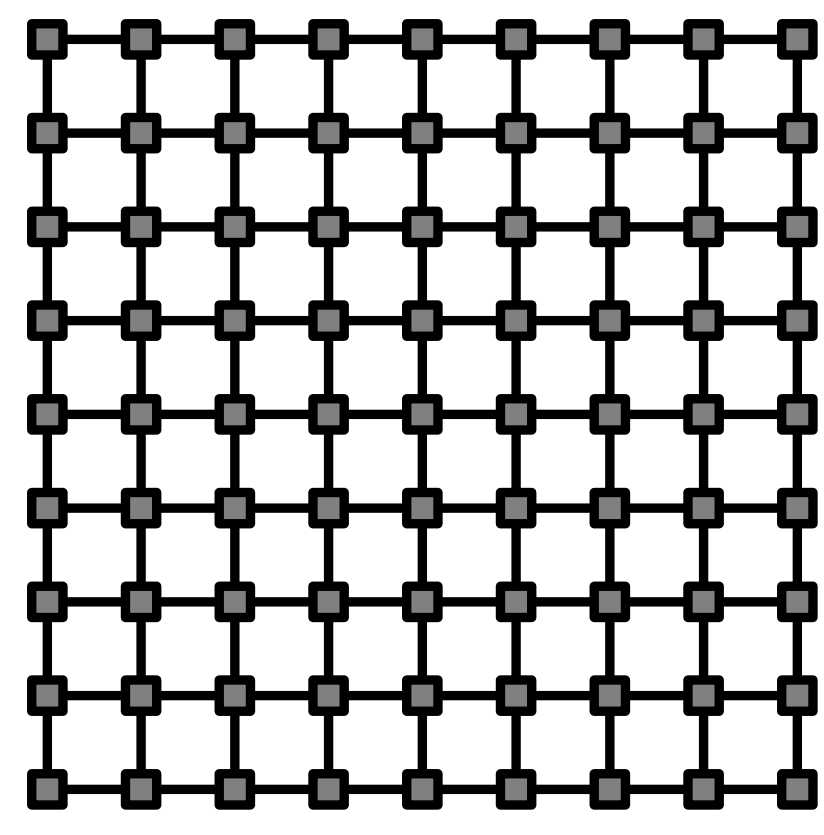

(a)

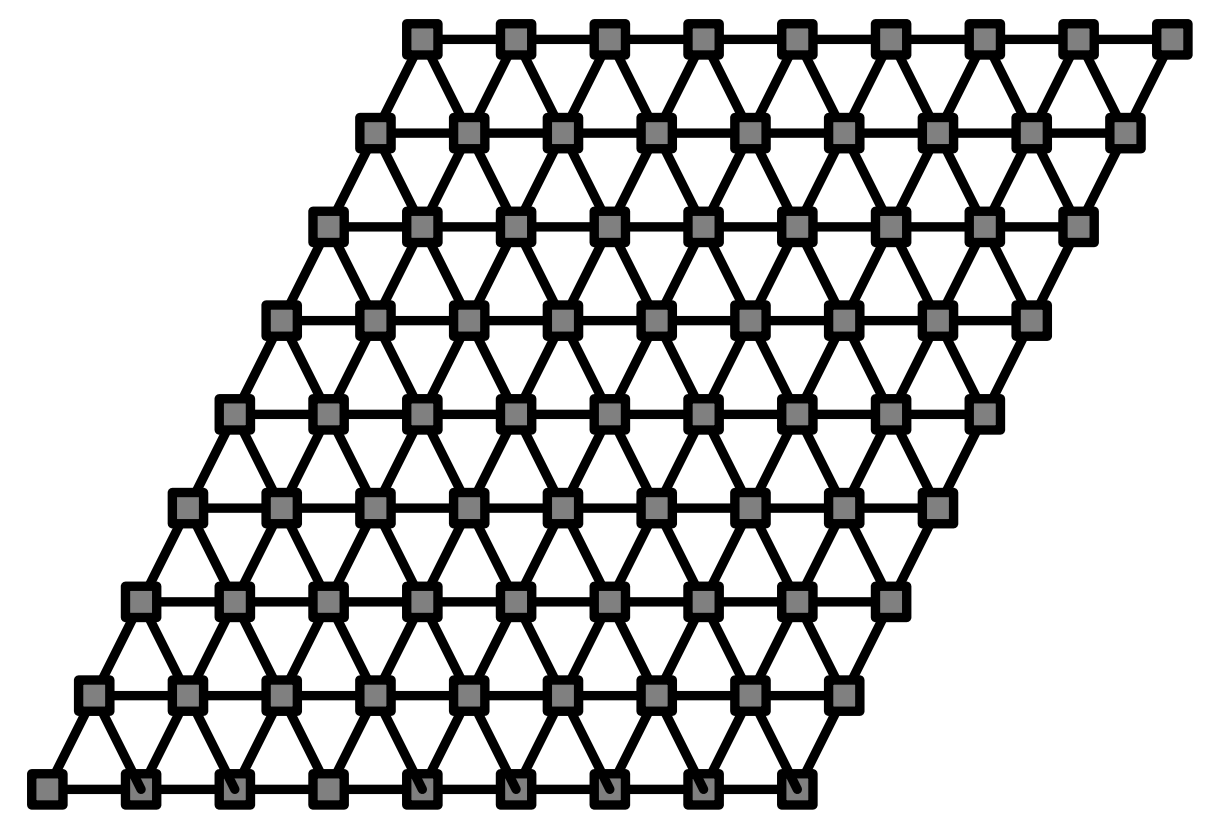

( b ) 


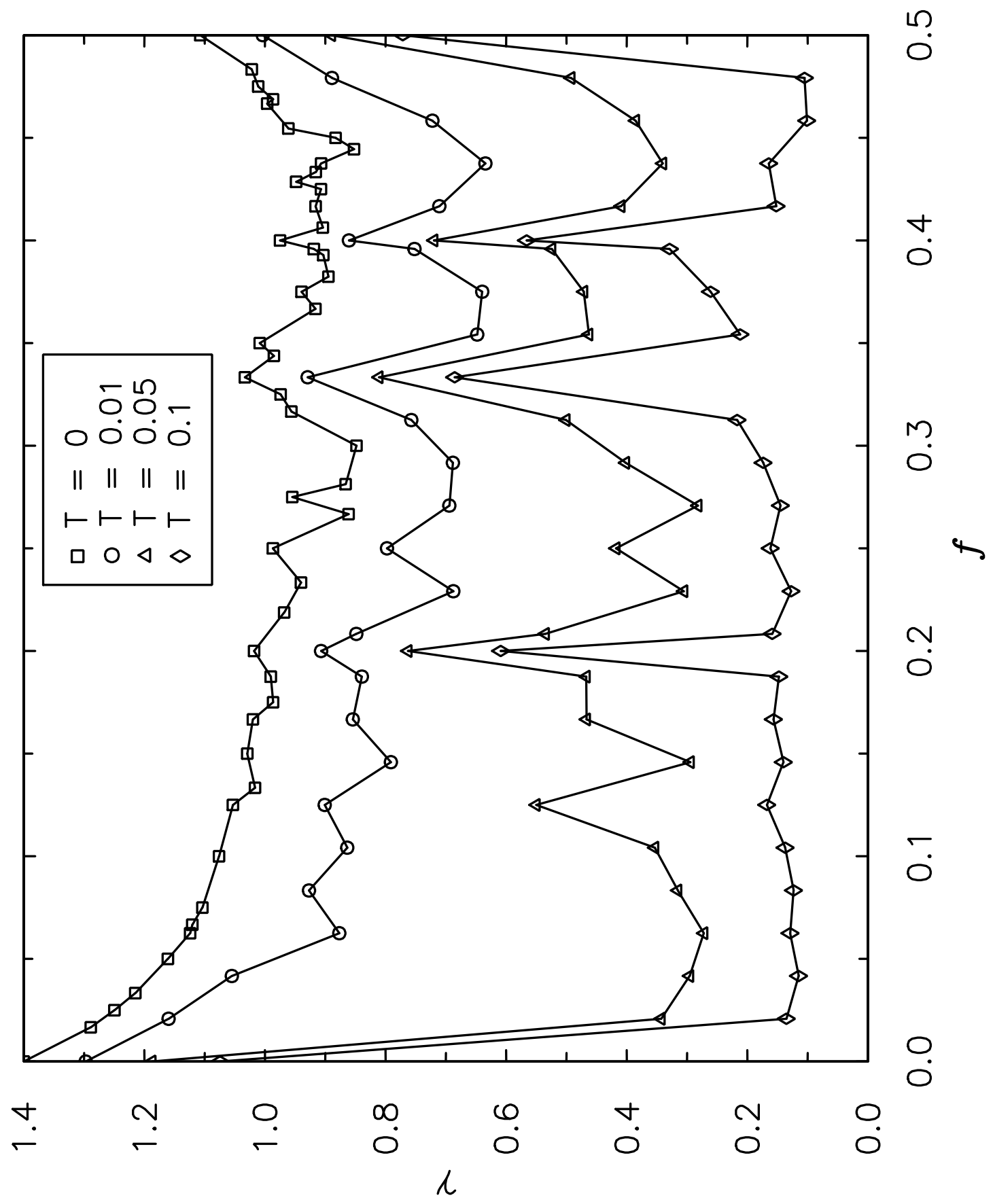




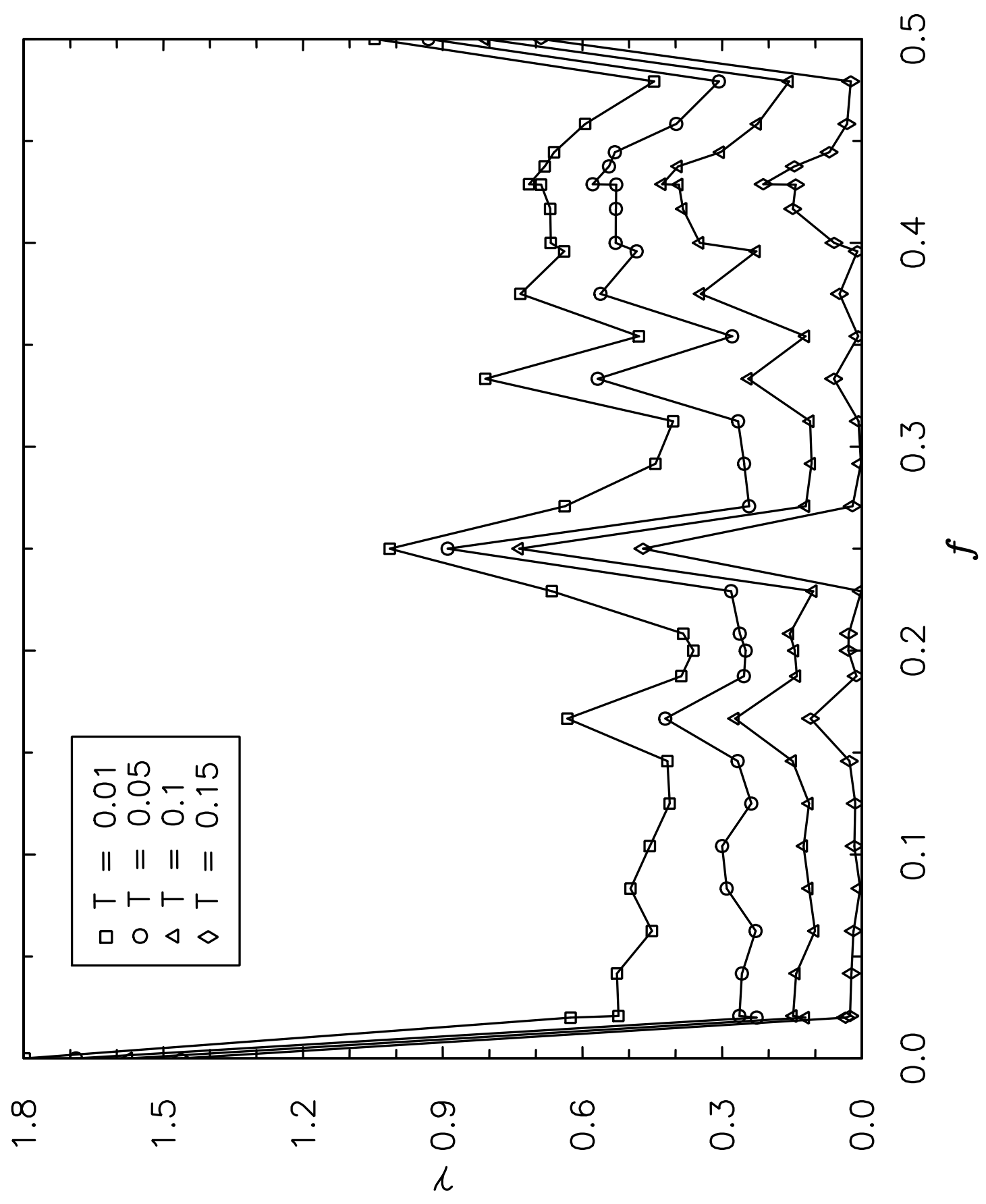




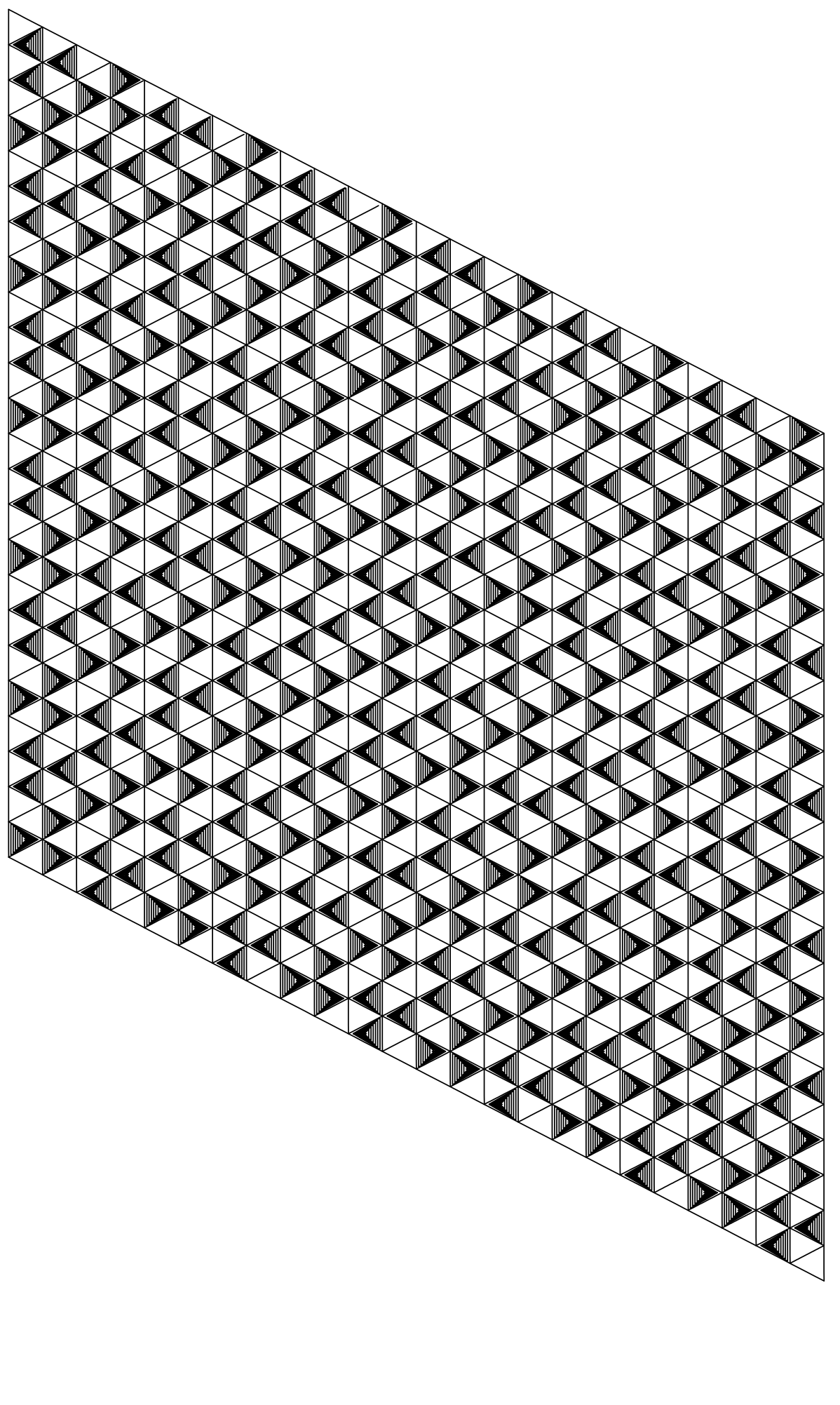





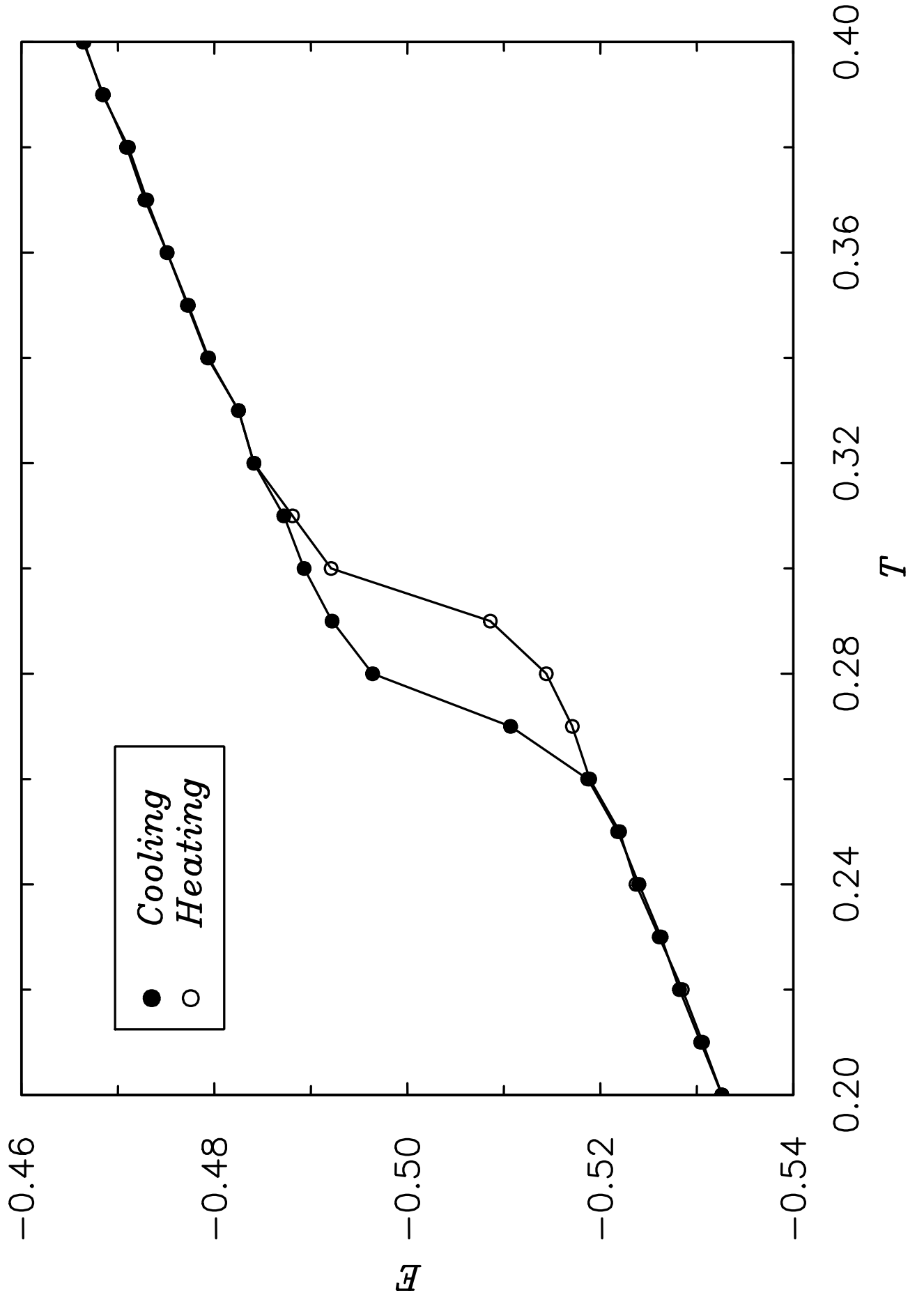

in 